\title{
Common fixed points and best proximity points of two cyclic self-mappings
}

M De la Sen ${ }^{1 *}$ and RP Agarwal2,3

${ }^{*}$ Correspondence:
manuel.delasen@ehu.es
${ }^{1}$ Instituto de Investigacion y
Desarrollo de Procesos, Universidad
del Pais Vasco, Campus of Leioa
(Bizkaia), Aptdo. 644, Bilbao, Bilbao
48080, Spain
Full list of author information is
available at the end of the article

available at the end of the article

\begin{abstract}
This paper discusses three contractive conditions for two 2-cyclic self-mappings defined on the union of two nonempty subsets of a metric space to itself. Such self-mappings are not assumed to commute. The properties of convergence of distances to the distance between such sets are investigated. The presence and uniqueness of common fixed points for the two self-mappings and the composite mapping are discussed for the case when such sets are nonempty and intersect. If the space is uniformly convex and the subsets are nonempty, closed and convex, then the iterates of points obtained through the self-mapping converge to unique best proximity points in each of the subsets. Those best proximity points coincide with the fixed point if such sets intersect.
\end{abstract}

\section{Introduction}

General rational contractive relations for self-mappings from certain sets into themselves have received important interest in the last years. The related background literature is very rich and, in particular, a very general rational contractive condition has been discussed in $[1,2]$. Relevant results about the existence of fixed points and their uniqueness under supplementary conditions have also been investigated in those papers. On the other hand, the rational contractive condition proposed in [3] is proved to include as particular cases several of the previously proposed ones [1,4-12], including Banach's principle [5] and Kannan's fixed point theorems $[4,8,9,11]$. Fixed point theory is also useful to investigate the stability of iterative sequences and discrete dynamic systems $[17,18,27]$. The rational contractive conditions of $[1,2]$ are applicable only on distinct points of the considered metric spaces. In particular, the fixed point theory for Kannan's mappings is extended in [4] by the use of a non-increasing function affecting the contractive condition and the best constant to ensure a fixed point is also obtained. Three fixed point theorems which extended the fixed point theory for Kannan's mappings have been stated and proved in [11]. Also, significant attention has been paid to the investigation of standard contractive and Meir-Keeler-type contractive 2-cyclic self-mappings $T: A \cup B \rightarrow A \cup B$ defined on subsets $A, B \subseteq X$ and, in general, $p$-cyclic self-mappings $T: \bigcup_{i \in \bar{p}} A_{i} \rightarrow \bigcup_{i \in \bar{p}} A_{i}$ defined on any number of subsets $A_{i} \subset X, i \in \bar{p}:=\{1,2, \ldots, p\}$, where $(X, d)$ is a metric space (see, for instance, [13-19] and [20-23]). More recent investigation of cyclic self-mappings has been devoted to its characterisation in partially ordered spaces and also to the formal extension of the contractive condition through the use of more general strictly increasing functions of the distance between adjacent subsets. In particular, the uniqueness of the

\section{黛 Springer}

(c) 2012 Sen and Agarwal; licensee Springer. This is an Open Access article distributed under the terms of the Creative Commons Attribution License (http://creativecommons.org/licenses/by/2.0), which permits unrestricted use, distribution, and reproduction in any medium, provided the original work is properly cited. 
best proximity points, to which all the sequences of iterates of composite self-mappings $T^{2}: A \cup B \rightarrow A \cup B$ converge, is proved in [14] for the extension of the contractive principle for cyclic self-mappings in uniformly convex Banach spaces (then being strictly convex and reflexive, [24]) if the subsets $A, B \subset X$ in the metric space $(X, d)$, or in the Banach space $(X,\|\|)$, where the 2 -cyclic self-mappings are defined are both nonempty, convex and closed. The research in [14] is centred on the case of the cyclic self-mapping being defined on the union of two subsets of the metric space. Those results have been extended in [15] for Meir-Keeler cyclic contraction maps and, in general, for the self-mapping $T$ : $\bigcup_{i \in \bar{p}} A_{i} \rightarrow \bigcup_{i \in \bar{p}} A_{i}$ be a $p(\geq 2)$-cyclic self-mapping being defined on any number of subsets of the metric space with $\bar{p}:=\{1,2, \ldots, p\}$.

A relevant problem is when self-mappings from a metric space into itself or from a set into itself have common fixed points, [19, 25-32]. A related problem is when composite self-maps built with those self-mappings have common fixed points with such selfmappings. There are some classical results available concerning the case when one of the self-mappings is continuous or when both self-mappings commute [25]. Some later extensions have removed the need for the continuity of one of the self-mappings [26, 28]. Some recent papers have investigated the existence of common fixed points in cone metric spaces $[29,30]$ and in fuzzy metric spaces and under contractive conditions of integral type [31,32]. This paper is concerned with the investigation of convergence properties of distances and the existence/uniqueness of common fixed points/common best proximity points of two 2-cyclic self-mappings (refereed to simply as cyclic self-mappings) on the union of two subsets $A$ and $B$ of a metric space under three contractive conditions. Section 2 is devoted to the convergence properties of distances for such contractive conditions which involve both cyclic self-mappings. Further results obtained in this section are concerned with the existence and uniqueness of common fixed points for the two cyclic self-mappings and their composite self-mapping if the involved subsets intersect and are closed and convex. Section 3 gives some direct extensions of the results in Section 2 when the most restrictive assumption in the section is removed. Finally, Section 4 extends the relevant results of the former sections to the case that $A$ and $B$ intersect in the sense that the role of common fixed points is played instead by common best proximity points under the assumption that the subsets $A$ and $B$ belong to a uniformly convex Banach space.

\section{Convergence properties and common fixed points under three contractive conditions if $A$ and $B$ intersect}

Let $(X, d)$ be metric space and consider two nonempty subsets $A$ and $B$ of $X$. It is assumed through the manuscript that $S, T: A \cup B \rightarrow A \cup B$ are cyclic self-mappings, i.e. $T(A) \subseteq B$, $S(A) \subseteq B, T(B) \subseteq A$ and $S(B) \subseteq A$. Suppose, in addition, that $T: A \cup B \rightarrow A \cup B$ satisfies the constraint

$$
\begin{aligned}
& d(S x, T S y) \leq \alpha d(x, S y)+\beta(d(x, S x)+d(S y, T S y))+\gamma(d(x, S y)+d(S x, T S y))+\omega D ; \\
& \forall x, y(\neq x) \in A \cup B,
\end{aligned}
$$

where

$$
D:=\operatorname{dist}(A, B):=\inf \{d(x, y): x \in A, y \in B\} \geq 0,
$$


where $\alpha \geq 0, \beta \geq 0, \gamma \geq 0, \omega \geq 0$. Note that if $x \in A$ and $y \in B$ or conversely, then the various point-to-point distances in (2.1) are not less than $D$ so that the parametrical constraint $\alpha+2(\beta+\gamma)+\omega \geq 1$ has to be fulfilled from (2.1) if $D \neq 0$. The following result can be stated:

Lemma 2.1 Assume that $d(x, S x) \leq d(x, T x) ; \forall x \in A \cup B$ and that the constraints $0 \leq 1$ $\omega \leq \alpha+2(\beta+\gamma)<1$ if $D \neq 0$ and $0 \leq \alpha+2(\beta+\gamma)<1$ if $D=0$ both hold. Then, the following properties hold:

(i)

$$
D \leq \limsup _{n \rightarrow \infty} d\left(S^{n} x, S^{n+1} x\right) \leq \frac{\omega D}{1-\alpha-2(\beta+\gamma)} ; \quad \forall x \in A \cup B .
$$

$$
\text { If } \omega=1-\alpha-2(\beta+\gamma) \text { then }
$$

$$
\exists \lim _{n \rightarrow \infty} d\left(S^{n} x, S^{n+1} x\right)=D
$$

(ii) $d\left(S^{n} x, S^{n+m+1} x\right) \leq \omega D+G_{m}(n)$ for any $x \in A \cup B, \forall n \in \boldsymbol{N}, \forall m \in \boldsymbol{N}_{0}=\boldsymbol{N} \cup\{0\}$, where $\left\{G_{m}(n)\right\}_{m \in \mathbf{N}_{0}}$ is a nonnegative strictly decreasing real sequence for any $n \in \boldsymbol{N}$, then being convergent to zero as $m \rightarrow \infty$, and

$$
\limsup _{m \rightarrow \infty} d\left(S^{n} x, S^{n+m+1} x\right) \leq \omega D
$$

Proof Take $y=x \in A \cup B$ and replace $x \rightarrow S^{n-1} x$ in (2.1) to yield

$$
\begin{aligned}
& d\left(S^{n} x, S^{n+1} x\right) \\
& \leq d\left(S^{n} x, T S^{n} x\right) \leq \alpha d\left(S^{n-1} x, S^{n} x\right)+\beta\left(d\left(S^{n-1} x, S^{n} x\right)+d\left(S^{n} x, T S^{n} x\right)\right) \\
&+\gamma\left(d\left(S^{n-1} x, S^{n} x\right)+d\left(T S^{n} x, S^{n} x\right)\right)+\omega D \\
& \leq \alpha d\left(S^{n-1} x, S^{n} x\right)+\beta\left(d\left(S^{n-1} x, S^{n} x\right)+d\left(S^{n} x, T S^{n} x\right)\right) \\
&+\gamma\left(d\left(S^{n-1} x, S^{n} x\right)+d\left(T S^{n} x, S^{n} x\right)\right)+\omega D ; \quad \forall x \in A \cup B, \forall n \in \boldsymbol{N}
\end{aligned}
$$

since $d(x, S x) \leq d(x, T x), S^{n} x \in A \Rightarrow\left(T S^{n} x \in B \wedge S^{n+1} x \in B\right)$ and $S^{n} x \in B \Rightarrow\left(T S^{n} x \in A \wedge\right.$ $S^{n+1} x \in A$ ) for any $x \in A \cup B$ and, equivalently,

$$
\begin{aligned}
d\left(S^{n} x, S^{n+1} x\right) & \leq d\left(S^{n} x, T S^{n} x\right) \\
& \leq \frac{\alpha+\beta+\gamma}{1-\beta-\gamma} d\left(S^{n-1} x, S^{n} x\right)+\frac{\omega D}{1-\beta-\gamma} ; \quad \forall x \in A \cup B, \forall n \in \boldsymbol{N}
\end{aligned}
$$

so that

$$
\begin{aligned}
D & \leq \limsup _{n \rightarrow \infty} d\left(S^{n} x, S^{n+1} x\right) \\
& \leq \lim _{n \rightarrow \infty}\left[\left(\frac{\alpha+\beta+\gamma}{1-\beta-\gamma}\right)^{n} d\left(S^{n-1} x, S^{n} x\right)+\left(\frac{\omega D}{1-\beta-\gamma}\right)\left(\sum_{i=1}^{n}\left(\frac{\alpha+\beta+\gamma}{1-\beta-\gamma}\right)^{n-i}\right)\right] \\
& \leq \frac{\omega D}{1-\alpha-2(\beta+\gamma)}
\end{aligned}
$$


since $0 \leq \beta+\gamma<1,1 \geq \omega \geq 1-\alpha-2(\beta+\gamma)$, and $0 \leq 1-\omega \leq \alpha+2(\beta+\gamma)<1$ if $D \neq 0$ and $0 \leq \alpha+2(\beta+\gamma)<1$ if $\bar{D}=0$ imply that the contraction constant $k:=\frac{\alpha+\beta+\gamma}{1-\beta-\gamma}<1$. Hence, (2.3) holds and the limit (2.4) exists for $\omega=1-\alpha-2(\beta+\gamma)$. Hence, Property (i) is proved. To prove Property (ii), note that for any natural numbers $m$ and $n$, one gets from (2.6) and the above definition of the contraction constant $k<1$ that

$$
\begin{aligned}
& d\left(S^{n} x, S^{n+m+1} x\right) \leq d\left(S^{n} x, T S^{n+m} x\right) \leq k^{m} d\left(S^{n+1} x, S^{n} x\right)+\omega D\left(1-k^{m}\right) \\
& \leq k^{m} d\left(S^{n} x, T S^{n} x\right)+\omega D\left(1-k^{m}\right) \\
& \leq d\left(S^{n} x, T S^{n} x\right)+\omega D ; \quad \forall x \in A \cup B, \forall n \in \mathbf{N}, \forall m \in \mathbf{N}_{0}, \\
& \limsup _{m \rightarrow \infty} d\left(S^{n} x, S^{n+m+1} x\right) \leq \omega D ; \quad \forall x \in A \cup B, \forall n \in \mathbf{N} .
\end{aligned}
$$

Hence, Property (ii) has been proved.

Note that the contractive condition

$$
d(S x, T S y) \leq \alpha d(x, S y)+\beta(d(x, S x)+d(S y, T S y))+\gamma d(x, S y)+\omega D
$$

is distinct from (2.1), while it modifies Lemma 2.1, resulting in the subsequent result.

Lemma 2.2 Assume that $d(x, S x) \leq d(x, T x) ; \forall x \in A \cup B$ and that the constraint $\omega \geq 1-$ $(\alpha+\gamma+2 \beta)>0$ holds. Then, the following properties hold:

(i)

$$
D \leq \limsup _{n \rightarrow \infty} d\left(S^{n} x, S^{n+1} x\right) \leq \frac{\omega D}{1-(\alpha+2 \beta+\gamma)} ; \quad \forall x \in A \cup B
$$

$$
\text { If } \omega=1-(\alpha+2 \beta+\gamma) \text { then }
$$

$$
\exists \lim _{n \rightarrow \infty} d\left(S^{n} x, S^{n+1} x\right)=D
$$

(ii) $d\left(S^{n} x, S^{n+m+1} x\right) \leq \omega D+F_{m}(n)$ for any $x \in A \cup B, \forall n \in \mathbf{N}, \forall m \in \boldsymbol{N}_{0}=\boldsymbol{N} \cup\{0\}$, where $\left\{F_{m}(n)\right\}_{m \in \mathbf{N}_{0}}$ is a nonnegative strictly decreasing real sequence for any $n \in \mathbf{N}$, then being convergent to zero as $m \rightarrow \infty$, and

$$
\limsup _{m \rightarrow \infty} d\left(S^{n} x, S^{n+m+1} x\right) \leq \omega D
$$

Proof The contractive condition (2.9) removes the additive term $d(S x, T S y)$ from (2.1) so that $d\left(T S^{n} x, S^{n} x\right)$ is correspondingly removed in the resulting modified counterpart of (2.5) by taking $y=x \in A \cup B$ and performing the replacement $x \rightarrow S^{n-1} x$. The resulting contractive constant now becomes $k:=\frac{\alpha+\beta}{1-\beta-\gamma}<1$, subject to $\frac{\omega D}{1-\beta-\gamma} \geq 1-\frac{\alpha+\beta}{1-\beta-\gamma}$, which, on the other hand, results in the needed constraint $1-\omega \leq \alpha+\gamma+2 \beta<1$ to reformulate the results of Lemma 2.1 leading to the modified Properties (i), with (2.10)-(2.11), and (ii).

The following two results are concerned with the existence and eventual uniqueness of fixed points of the self-mappings $S, T, S, T \circ S: A \cup B \rightarrow A \cup B$ (with $(T \circ S) x=T S x$, $\forall x \in A \cup B)$ if $A$ and $B$ intersect, are nonempty and closed. If they are also convex then the fixed point is unique, fulfilling the property $\operatorname{Fix}(S) \equiv \operatorname{Fix}(T) \equiv \operatorname{Fix}(T \circ S)=\{z\} \subset A \cap B$. 
Theorem 2.3 Let $(X, d)$ be a complete metric space and assume that $S, T: A \cup B \rightarrow A \cup B$ are cyclic self-mappings, where $A$ and $B$ intersect, are nonempty and closed, and that the contractive condition (2.1) holds subject to $0 \leq \alpha+2(\beta+\gamma)<1$. Then, there exists a fixed point of $S: A \cup B \rightarrow A \cup B$ in $A \cap B$ which is also a fixed point of the composite self-mapping $T \circ S: A \cup B \rightarrow A \cup B$ and a fixed point of $T: A \cup B \rightarrow A \cup B$. If, in addition, $A$ and $B$ are convex, then $\operatorname{Fix}(S) \equiv \operatorname{Fix}(T \circ S) \equiv \operatorname{Fix}(T)$ consists of a single point.

Proof From Lemma 2.1 and $D=0$ (since $A \cap B \neq \emptyset$ ), it follows that

$$
\lim _{n \rightarrow \infty} d\left(S^{n} x, S^{n+1} x\right)=\lim _{n \rightarrow \infty} d\left(S^{n} x, T S^{n} x\right)=0 ; \quad \forall x \in A \cup B
$$

Thus, $\lim _{n, m \rightarrow \infty} d\left(S^{n+m} x, S^{n+m+1} x\right)=\lim _{n, m \rightarrow \infty} d\left(S^{n+m} x, T S^{n+m} x\right)=0 ; \forall x \in A \cup B$ so that $\left\{S^{n} x\right\}_{n \in \boldsymbol{N}}$ is a Cauchy sequence; $\forall x \in A \cup B$, then it is convergent to some $z \in A \cap B$ since $A \cap B$ is nonempty and closed. Also, since $S: A \cup B \rightarrow A \cup B$ is contractive from Lemma 2.1, then it is globally Lipschitz-continuous for any pair $(x, S x)$ with $x \in$ $A \cup B$ and then $\left\{S^{n} x\right\}_{n \in \boldsymbol{N}}$ is in $A \cap B$. Thus, $S^{n+1} x=S\left(S^{n} x\right) \rightarrow z=S z ; \forall x \in A \cup B$ and $z \in \operatorname{Fix}(S) \subset A \cap B$. Since $S^{n} x \rightarrow z$ and $\lim _{n \rightarrow \infty} d\left(S^{n} x, T S^{n} x\right)=0 ; \forall x \in A \cup B$ then $T S^{n+1} x \rightarrow T S^{n} x \rightarrow z \Rightarrow(T S)\left(S^{n} x\right) \rightarrow T S z=z$. Thus, $z \in \operatorname{Fix}(S) \cap \operatorname{Fix}(T \circ S) \subset A \cap B$ and $T S z=S z=z \Rightarrow T^{2} S z=T(T S z)=T S z=z=T z$. Then, $z \in \operatorname{Fix}(S) \cap \operatorname{Fix}(T) \cap \operatorname{Fix}(T \circ S) \subset$ $A \cap B$.

Finally assume, in addition, that $A$ and $B$ are also convex and $z_{1}, z_{2}\left(\neq z_{1}\right) \in \operatorname{Fix}(S \circ T) \cap$ $\operatorname{Fix}(S) \cap \operatorname{Fix}(T)$ so that

$$
z_{1}, z_{2}, \quad S^{n} z_{1}=z_{1}, \quad T S^{n} z_{2}=z_{2} \in \operatorname{Fix}(T \circ S) \cap \operatorname{Fix}(S) \subset A \cap B
$$

since $A \cap B$ is convex. Using (2.1) with $x=z_{1}, y=z_{2}$ and $D=0$, the following contradictions lead to $z_{1} \neq z_{2}$ from the contractive assumption in Lemma 2.1 since $0 \leq \alpha<1-2(\beta+\gamma)<1$ :

$$
\begin{aligned}
& 0<d\left(z_{1}, z_{2}\right) \leq \alpha^{n} d\left(z_{1}, z_{2}\right)<d\left(z_{1}, z_{2}\right) ; \quad \forall n \in \boldsymbol{N}, \\
& 0<d\left(z_{1}, z_{2}\right) \leq \lim _{n \rightarrow \infty}\left(\alpha^{n}\right) d\left(z_{1}, z_{2}\right)=0
\end{aligned}
$$

so that $z_{1}=z_{2}$ and $\operatorname{Fix}(S) \equiv \operatorname{Fix}(T) \equiv \operatorname{Fix}(T \circ S)=\{z\} \subset A \cap B$. Hence, the proof is complete.

Theorem 2.4 Theorem 2.3 applies "mutatis-mutandis" for the contractive constraint (2.9) subject to $\omega \geq 1-(\alpha+\gamma+2 \beta)>0$.

The proof of Theorem 2.4 is omitted since it is similar to that of Theorem 2.3.

Assume now that the contractive condition (2.1) is modified as follows to give relevance to the composite self-mapping $S \circ T: A \cup B \rightarrow A \cup B$ :

$$
\begin{aligned}
& \max \left(\mu^{-1} d\left(T x, T^{2} x\right), v^{-1} d\left(S x, S^{2} x\right)\right) \\
& \quad \leq d(S x, S T x) \\
& \quad \leq \alpha d(x, T x)+\beta d\left(T x, T^{2} x\right)+\delta d(x, S x)+\gamma d\left(S x, S^{2} x\right)+\omega D ; \quad \forall x \in A \cup B
\end{aligned}
$$


for some real constants $\alpha, \beta, \delta, \gamma \geq 0$ and $\mu, \nu \geq 0$ so that by using the lower-bound of (2.13) to build a further upper-bounding condition of it, one gets

$$
\begin{aligned}
d(S x, S T x) \leq & \alpha d(x, T x)+\beta d\left(T x, T^{2} x\right)+\delta d(x, S x)+\gamma d\left(S x, S^{2} x\right)+\omega D \\
\leq & \alpha d(x, T x)+\beta \mu d(S x, S T x)+\delta \nu d(x, T x) \\
& +\gamma \nu d(S x, S T x)+\omega D ; \quad \forall x \in A \cup B,
\end{aligned}
$$

which is identical to

$$
d(S x, S T x) \leq \frac{\alpha+\delta}{1-\beta \mu-\gamma \nu} d(x, T x)+\frac{\omega D}{1-\beta \mu-\gamma \nu} ; \quad \forall x \in A \cup B
$$

if $\alpha+\delta+\beta \mu+\gamma \nu<1$. The following two results hold under the contractive condition (2.12).

Lemma 2.5 Assume that the contractive condition (2.12) holds subject to $\alpha+\delta+\beta \mu+\gamma \nu<1$ and $\omega \geq 1-\alpha-2(\beta+\gamma)$. Then

$$
\begin{aligned}
& d\left(S^{n} x, S^{n} T x\right) \leq k^{n} d(x, T x)+\frac{\omega D}{1-\beta \mu-\gamma \nu}\left(1-k^{n}\right) \\
& \leq d(x, T x)+\frac{\omega D}{1-\beta \mu-\gamma \nu} ; \quad \forall x \in A \cup B, \forall n \in \mathbf{N}, \\
& \limsup _{n \rightarrow \infty} d\left(S^{n} x, S^{n} T x\right) \leq \frac{\omega D}{1-\beta \mu-\gamma \nu} ; \quad \forall x \in A \cup B
\end{aligned}
$$

and

$$
\exists \lim _{n \rightarrow \infty} d\left(S^{n} x, S^{n} T x\right)=D ; \quad \forall x \in A \cup B
$$

Proof Redefine the contractive constant as $k:=\frac{\alpha+\delta}{1-\beta \mu-\gamma \nu}<1$ so that $\frac{\omega D}{1-\beta \mu-\gamma \nu} \geq 1-k$ if $\omega \geq$ $1-\beta \mu-\gamma \nu-\alpha-\delta$. One gets (2.15)-(2.17) directly from (2.14).

Theorem 2.6 Let $(X,\|\|)$ be a Banach space and assume that $S, T: A \cup B \rightarrow A \cup B$ are cyclic self-mappings satisfying the contractive condition (2.12) subject to $\alpha+\delta+\beta \mu+\gamma \nu<1$, where $A$ and $B$ intersect and are nonempty and closed. Then, there exists a fixed point of $S: A \cup B \rightarrow A \cup B$ in $A \cap B$ which is also a fixed point of the composite self-mapping $T \circ S: A \cup B \rightarrow A \cup B$. If, in addition, $A$ and $B$ are convex then $\operatorname{Fix}(S) \equiv \operatorname{Fix}(T \circ S)$ consists of a single point.

Outline of proof Let $(X, d)$ be the complete metric space where $d: X \times X \rightarrow \boldsymbol{R}_{0+}$ is the norm-induced metric by the norm on the Banach space $(X,\|\|)$. If $A$ and $B$ intersect, then $\lim _{n \rightarrow \infty} d\left(S^{n} x, S^{n} T x\right)=0 ; \forall x \in A \cup B$ from Lemma 2.5 with $S: A \cup B \rightarrow A \cup B$ satisfying a contractive condition and then being globally Lipschitz continuous for any pair $(x, T x)$ with $x \in A \cup B$. Thus, the following general terms of Cauchy sequences converge to a fixed point; i.e. $S^{n+1} x=S\left(S^{n} x\right), S^{n} x, S^{n} T x \rightarrow z$ so that $z \in \operatorname{Fix}(S) \equiv \operatorname{Fix}(S \circ T) \subset A \cap B$. The uniqueness of the fixed point is proved by using the convexity of $A \cap B$ as follows. Assume 
that $z, z_{1}(\neq z)$ are fixed points of $S, S \circ T: A \cup B \rightarrow A \cup B$ in $A \cap B$. Then, the following contradictions lead to $z \neq z_{1}$ in terms that either

$$
\begin{aligned}
0 & <\left\|S\left(\frac{z+z_{1}}{2}\right)-S T\left(\frac{z+z_{1}}{2}\right)\right\|=d\left(S \frac{z+z_{1}}{2}, S T \frac{z+z_{1}}{2}\right)=\left\|\frac{S T z-S z}{2}-\frac{S T z_{1}-S z_{1}}{2}\right\| \\
& \leq \frac{1}{2}\|S T z-S z\|+\frac{1}{2}\left\|S T z_{1}-S z_{1}\right\|=\frac{1}{2}\left(d(S z, S T z)+d\left(S z_{1}, S T z_{1}\right)\right)=0
\end{aligned}
$$

since $\frac{z+z_{1}}{2} \in A \cap B$, since $A \cap B$ is convex, or all points in the segment $\left[z, z_{1}\right] \subset A \cap B$, again since $A \cap B$ is convex, are fixed points of the self-mappings $S, S \circ T: A \cup B \rightarrow A \cup B$. Now, take arbitrarily closed points $z^{\prime}, z^{\prime \prime}=z^{\prime+} \in\left(z, z_{1}\right)$, which are also fixed points. Then the continuity of $S: A \cup B \rightarrow A \cup B$ leads to a further contradiction $\lim _{z^{\prime} \rightarrow z^{\prime \prime}}\left(S z^{\prime}\right)=z^{\prime \prime} \neq z^{\prime}$. Then, no segment $\left[z, z_{1}\right] \subset A \cap B$ can consist of fixed points of $S, S \circ T: A \cup B \rightarrow A \cup B$.

\section{Relaxing a hypothesis of Section 2}

The assumption $d(x, S x) \leq d(x, T x) ; \forall x \in A \cup B$ in Lemma 2.1 and Lemma 2.2, then in Theorem 2.3 and Theorem 2.4, can be removed at the expense of more restrictive constraints on the corresponding contractive conditions on the parameters. For instance, the triangle inequality for distances yields

$$
d\left(S^{n} x, S^{n+1} x\right) \leq d\left(S^{n} x, T S^{n} x\right)+d\left(T S^{n} x, S^{n+1} x\right) ; \quad \forall x \in A \cup B .
$$

The contractive condition (2.1) becomes equivalent to

$$
\begin{aligned}
d\left(S^{n+1} x, T S^{n} x\right) \leq & \beta\left(d\left(S^{n} x, S^{n+1} x\right)+d\left(S^{n} x, T S^{n} x\right)\right) \\
& +\gamma d\left(S^{n+1} x, T S^{n} x\right)+\omega D ; \quad \forall x \in A \cup B
\end{aligned}
$$

with the replacements $x, S y \rightarrow S^{n} x, y \rightarrow S^{n-1} x$. The inequality (3.2) is equivalent to

$$
d\left(S^{n+1} x, T S^{n} x\right) \leq \frac{\beta}{1-\gamma}\left(d\left(S^{n} x, S^{n+1} x\right)+d\left(S^{n} x, T S^{n} x\right)\right)+\frac{\omega D}{1-\gamma} ; \quad \forall x \in A \cup B
$$

if $0 \leq \gamma<1$. The substitution of (3.3) into (3.1) yields

$$
\begin{aligned}
d\left(S^{n} x, S^{n+1} x\right) \leq & \frac{\beta}{1-\gamma}\left(d\left(S^{n} x, S^{n+1} x\right)+d\left(S^{n} x, T S^{n} x\right)\right) \\
& +d\left(S^{n} x, T S^{n} x\right)+\frac{\omega D}{1-\gamma} ; \quad \forall x \in A \cup B
\end{aligned}
$$

and using (2.6) in (3.4)

$$
\begin{aligned}
& d\left(S^{n+1} x, S^{n} x\right) \\
& \quad \leq \frac{1+\beta-\gamma}{1-\beta-\gamma} d\left(S^{n} x, T S^{n} x\right)+\frac{\omega D}{1-\beta-\gamma} \\
& \leq \frac{1+\beta-\gamma}{1-\beta-\gamma}\left(\frac{\alpha+\beta+\gamma}{1-\beta-\gamma} d\left(S^{n-1} x, S^{n} x\right)+\frac{\omega D}{1-\beta-\gamma}\right)+\frac{\omega D}{1-\beta-\gamma} \\
& \quad=\frac{(1+\beta-\gamma)(\alpha+\beta+\gamma)}{(1-\beta-\gamma)^{2}} d\left(S^{n-1} x, S^{n} x\right)+\frac{2(1-\gamma) \omega D}{(1-\beta-\gamma)^{2}} ; \quad \forall x \in A \cup B
\end{aligned}
$$


and $S: A \cup B \rightarrow A \cup B$ is cyclic contractive if $\frac{(1+\beta-\gamma)(\alpha+\beta+\gamma)}{(1-\beta-\gamma)^{2}}<1$, that is, if

$$
\begin{aligned}
& 0 \leq \alpha<\frac{(1-\beta-\gamma)^{2}}{1+\beta-\gamma}-(\beta+\gamma) ; \quad 0 \leq \beta<\frac{1+\gamma(2 \gamma-3)}{3-2 \gamma} ; \quad \gamma \in(1 / 2,1), \\
& \omega \geq \frac{(1-\beta-\gamma)^{2}-(1+\beta-\gamma)(\alpha+\beta-\gamma)}{2(1-\gamma)},
\end{aligned}
$$

where the second constraint of (3.6) guarantees that $\beta+\gamma<\frac{(1-\beta-\gamma)^{2}}{1+\beta-\gamma}$; i.e. $\alpha \geq 0$ and the third one that $\beta$ is nonnegative. Lemma 2.1(i) is modified by using (3.5)-(3.7) as follows without using the assumption $d(x, S x) \leq d(x, T x) ; \forall x \in A \cup B$.

Lemma 3.1 Assume that (2.1) holds subject to the constraints (3.6)-(3.7). Then

$$
\begin{aligned}
& D \leq \limsup _{n \rightarrow \infty} d\left(S^{n} x, S^{n+1} x\right) \leq \frac{2(1-\gamma) \omega D}{(1-\beta-\gamma)^{2}} ; \quad \forall x \in A \cup B . \\
& \text { If } \omega=\frac{(1-\beta-\gamma)^{2}-(1+\beta-\gamma)(\alpha+\beta-\gamma)}{2(1-\gamma)}, \text { then } \\
& \exists \lim _{n \rightarrow \infty} d\left(S^{n} x, S^{n+1} x\right)=D .
\end{aligned}
$$

An "ad-hoc" modified version of Theorem 2.3 follows.

Theorem 3.2 Let $(X, d)$ be a complete metric space and assume that $S, T: A \cup B \rightarrow A \cup B$ are cyclic self-mappings where $A$ and $B$ intersect and are nonempty and closed, and that the contractive condition (2.1) holds subject to

$$
\begin{aligned}
& 0 \leq \alpha<\frac{(1-\beta-\gamma)^{2}}{1+\beta-\gamma}-(\beta+\gamma) ; \quad 0 \leq \beta<\frac{1+\gamma(2 \gamma-3)}{3-2 \gamma} ; \quad \gamma \in(1 / 2,1), \\
& \omega=\frac{(1-\beta-\gamma)^{2}-(1+\beta-\gamma)(\alpha+\beta-\gamma)}{2(1-\gamma)} .
\end{aligned}
$$

Then, there exists a fixed point $z$ of $S: A \cup B \rightarrow A \cup B$ in $A \cap B$. If, in addition, $0 \leq \beta<1-2 \gamma$ and $0 \leq \gamma \leq 1 / 2$ then $z$ is a fixed point of the composite self-mapping $T \circ S: A \cup B \rightarrow A \cup B$ and also of the self-mapping $T: A \cup B \rightarrow A \cup B$. If, furthermore, $A$ and $B$ are convex, then $\operatorname{Fix}(S) \equiv \operatorname{Fix}(T \circ S) \equiv \operatorname{Fix}(T)$ consists of a single point.

Proof It follows from Lemma 3.1 that if $D=0$ then $\left\{S^{n} x\right\}_{n \in N}$ is a Cauchy sequence convergent in the closed set $A \cap B$ since $S: A \cup B \rightarrow A \cup B$ is globally Lipschitz continuous for any $x \in A \cup B$ from (3.9). Thus, $\exists z \in \operatorname{Fix}(S) \subset A \cap B$, which satisfies $S^{n} x \rightarrow z=S z$ for any given $x \in A \cup B$. If, in addition, we take $x=y=z=S z$ and $D=0$ in (2.1) with $0 \leq \beta<1-2 \gamma$ and $0 \leq \gamma \leq 1 / 2$, one gets

$$
d(S z, T S z)=d(S z, T z) \leq \frac{\gamma}{1-\beta-\gamma} d(z, S z)
$$

which only holds if and only if $S z=T S z=T z=z$ so that $z \in \operatorname{Fix}(S) \cap \operatorname{Fix}(T \circ S) \cap \operatorname{Fix}(T) \subset$ $A \cap B$. The uniqueness of the fixed point follows as in the proof of Theorem 2.3 by using the convexity assumption. 
Reformulations of Lemma 2.2 and Theorem 2.4 without using $d(x, S x) \leq d(x, T x) ; \forall x \in$ $A \cup B$ could be made in a similar way.

\section{Properties of convergence and common best proximity points for the case when $A$ and $B$ do not intersect}

This section extends some relevant results from the previous sections to the case that the subsets do not intersect provided they are subsets of a uniformly convex Banach space. For such a case, Lemmas 2.1, 2.2, 2.5 and 3.1 still hold. However, Theorems 2.3, 2.4, 2.6 and 3.2 do not further hold since fixed points in $A \cap B=\emptyset$ cannot exist. Thus, the further investigation is centred on the existence and potential uniqueness of best proximity points. It has been proved in [1] that if $T: A \cup B \rightarrow A \cup B$ is a cyclic $\varphi$-contraction with $A$ and $B$ being weakly closed subsets of a reflexive Banach space $(X,\|\|)$, then $\exists(x, y) \in A \times B$ such that $D=d(x, y)=\|x-y\|$ where $d: \boldsymbol{R}_{0_{+}} \rightarrow \boldsymbol{R}_{0+}$ is a norm-induced metric, i.e. $x$ and $y$ are best proximity points. Also, if $A$ and $B$ are nonempty subsets of a metric space $(X, d), A$ is compact, $B$ is approximatively compact with respect to $A$ and $T: A \cup B \rightarrow A \cup B$ is a cyclic contraction, then $\exists(x, y) \in A \times B$ such that $D=d(x, y)\left(\right.$ i.e. if $\lim _{n \rightarrow \infty} d\left(T^{2 n} x, y\right)=d(B, y):=$ $\inf _{z \in B} d(z, y)$ for some $y \in A$ and all $x \in B$ then the sequence $\left\{T^{2 n} x\right\}_{n \in N_{0}}$ has a convergent subsequence, [14]). Theorem 2.3 extends as follows, via Lemma 2.1, for the general case when $A$ and $B$ do not intersect.

Theorem 4.1 Assume that $A$ and $B$ are nonempty closed and convex subsets of $a$ uniformly convex Banach space $(X,\|\|)$. Assume also that $S, T: A \cup B \rightarrow A \cup B$ are both cyclic selfmappings and that the contractive condition (2.1) holds subject to $\min (\alpha, \beta, \gamma) \geq 0, \alpha+2(\beta+$ $\gamma)<1$ and $d(x, S x) \leq d(x, T x) ; \forall x \in A \cup B$. Then, there exist two unique best proximity points $z \in A, y \in B$ of the self-mappings $S, T, T \circ S: A \cup B \rightarrow A \cup B$ such that

$$
\begin{aligned}
& z=S y=T y=T S^{2} y=T S z=S^{2} z=S^{2} T y=S^{3} y, \\
& y=S z=T z=T S^{2} z=T S y=S^{2} y=S^{2} T z=S^{3} z .
\end{aligned}
$$

If $A \cap B \neq \emptyset$, then $z=y \in A \cap B$ is the unique fixed point of $S, T, T \circ S: A \cup B \rightarrow A \cup B$ which is in $A \cap B$.

Proof If $D=0$, i.e. $A$ and $B$ intersect, then this result reduces to Theorem 2.3, with the best proximity points being coincident and equal to the unique fixed point. Consider the case that $A$ and $B$ do not intersect, that is, $D>0$, and take $x \in A \cup B$. Assume that $x \in A$. Since $A$ and $B$ are nonempty and closed, $A$ is convex and Lemma 2.1(i) holds; $\operatorname{since} \min (\alpha, \beta, \gamma) \geq$ $0, \alpha+2(\beta+\gamma)<1$ and $d(x, S x) \leq d(x, T x) ; \forall x \in A \cup B$, it follows that

$$
\begin{aligned}
& {\left[d\left(S^{2 n+1} x, S^{2 n} x\right) \rightarrow D ; d\left(S^{2 n+1} x, S^{2 n+2} x\right) \rightarrow D\right]} \\
& \quad \Rightarrow \quad d\left(S^{2 n} x, S^{2 n} x\right) \rightarrow 0 \quad \text { as } n \rightarrow \infty
\end{aligned}
$$

(which was proved in Lemma 3.8 [14]). The same conclusion arises if $x \in B$ since B is convex. Thus, $\left\{S^{2 n} x\right\}_{n \in \boldsymbol{N}_{0}}$ is bounded and converges to some point $z=z(x)$, being potentially dependent on the initial point $x$ which is in $A$ if $x \in A$, since $A$ is closed, and in $B$ if $x \in B$, since $B$ is closed. Take, with no loss in generality, the norm-induced metric and 
consider the associate metric space $(X, d)$ which can be identified with $(X,\|\|)$ in this context. It is now proved by contradiction that for every $\varepsilon \in \boldsymbol{R}_{+}$, there exists $n_{0} \in \boldsymbol{N}_{0}$ such that $d\left(S^{2 m} x, S^{2 n+1} x\right) \leq D+\varepsilon$ for all $m>n \geq n_{0}$. Assume the contrary; that is, given some $\varepsilon \in \boldsymbol{R}_{+}$, there exists $n_{0} \in \boldsymbol{N}_{0}$ such that $d\left(S^{2 m_{k}} x, S^{2 n_{k}+1} x\right)>D+\varepsilon$ for all $m_{k}>n_{k} \geq n_{0}, \forall k \in \boldsymbol{N}_{0}$. Then, by using the triangle inequality for distances

$$
\begin{aligned}
D+\varepsilon & <d\left(S^{2 m_{k}} x, S^{2 n_{k}+1} x\right) \\
& \leq d\left(S^{2 m_{k}} x, S^{2 m_{k}+2} x\right)+d\left(S^{2 m_{k}+2} x, S^{2 n_{k}+1} x\right) \quad \text { as } n \rightarrow \infty
\end{aligned}
$$

one gets from (3.1)-(3.2) that

$$
\begin{gathered}
\liminf _{k \rightarrow \infty}\left(d\left(S^{2 m_{k}} x, S^{2 m_{k}+2} x\right)+d\left(S^{2 m_{k}+2} x, S^{2 n_{k}+1} x\right)\right) \\
\quad=\liminf _{k \rightarrow \infty} d\left(S^{2 m_{k}+2} x, S^{2 n_{k}+1} x\right)>D+\varepsilon .
\end{gathered}
$$

Now, one gets from (4.3), (4.5) and Lemma 2.1(i) the following contradiction:

$$
\begin{aligned}
D+\varepsilon & <\limsup _{k \rightarrow \infty} d\left(S^{2 m_{k}+2} x, S^{2 n_{k}+1} x\right) \\
& \leq \limsup _{n_{k} \rightarrow \infty} d\left(S^{2 n_{k}+2} x, S^{2 n_{k}+1} x\right)+\limsup _{k \rightarrow \infty} d\left(S^{2 m_{k}+2} x, S^{2 n_{k}+2} x\right) \\
& =\limsup _{n_{k} \rightarrow \infty} d\left(S^{2 n_{k}+2} x, S^{2 n_{k}+1} x\right)=D .
\end{aligned}
$$

As a result, $d\left(S^{2 m} x, S^{2 n+1} x\right) \leq D+\varepsilon$ for every given $\varepsilon \in \boldsymbol{R}_{+}$and all $m>n \geq n_{0}$ for some existing $n_{0} \in \boldsymbol{N}_{0}$. This leads by a choice of arbitrarily small $\varepsilon$ to

$$
D \leq \limsup _{n \rightarrow \infty} d\left(S^{2 m} x, S^{2 n+1} x\right) \leq D \quad \Rightarrow \quad \exists \lim _{n \rightarrow \infty} d\left(S^{2 m} x, S^{2 n+1} x\right)=D .
$$

But $\left\{S^{2 n} x\right\}_{n \in \boldsymbol{N}_{0}}$ is a Cauchy sequence with a limit $z=S^{2} z$ in $A$ (respectively, with a limit $y=$ $S^{2} y$ in $B$ ) if $x \in A$ (respectively, if $x \in B$ ) such that $D=\|S z-z\|=d(z, S z)$ (Proposition 3.2, [14]). Assume on the contrary that $x \in A$ and $\left\{S^{2 n} x\right\}_{n \in N_{0}} \rightarrow z \neq S^{2} z$ as $n \rightarrow \infty$ so that $S^{2} z-S z=z-S z \neq z-y$; so that since $A$ is convex and $(X,\|\|)$ is a uniformly convex Banach space, then being strictly convex, one has

$$
\begin{aligned}
D & =d(z, S z)=d\left(\frac{S^{2} z+z}{2}-S z\right)=\left\|\frac{S^{2} z-S z}{2}+\frac{z-S z}{2}\right\| \\
& \leq\left\|\frac{S^{2} z-S z}{2}\right\|+\left\|\frac{z-S z}{2}\right\|<\frac{D}{2}+\frac{D}{2}=D,
\end{aligned}
$$

which is a contradiction and $z=S^{2} z$ is the best proximity point in $A$ of $S: A \cup B \rightarrow A \cup B$. In the same way, $\left\{S^{2 n} x\right\}_{n \in N_{0}}$ is a Cauchy sequence with a limit $S^{2} y=y \in B$ which is the best proximity point in $B$ of $S: A \cup B \rightarrow A \cup B$ if $x \in B$ since $B$ is convex and $(X,\|\|)$ is strictly convex. We prove now that $y=S z$. Assume, on the contrary, that $y \neq S z$ with $y=S^{2} y, S z=S^{3} z \in B, z=S^{2} z \in A, d(z, y)>D, d(S z, S y) \geq D, d(S z, z)=d(S y, y)=D$. One gets the following contradiction from (2.5), which is obtained from (2.1) provided that $d(x, S x) \leq d(x, T x) ; \forall x \in A \cup B$, since $S: A \cup B \rightarrow A \cup B$ is globally Lipschitz continuous 
from Banach contraction principle since all composite self-mappings $S^{2 n}: A \cup B \rightarrow A \cup B$; $n \in \boldsymbol{N}$ are contractive:

$$
\begin{aligned}
& {\left[d\left(S^{n} x, S^{n+1} x\right) \rightarrow D=d(z, y) ; \forall x \in A \cup B \text { as } n \rightarrow \infty\right]} \\
& \quad \Rightarrow \quad\left[y \leftarrow S^{n+1} x=S\left(S^{n} x\right) \rightarrow S z \text { as } n \rightarrow \infty\right] .
\end{aligned}
$$

Thus, $z=S y=S^{2} z=S^{3} y$ and $y=S z=S^{2} y=S^{3} z$ are the best proximity points of $S$ : $A \cup B \rightarrow A \cup B$ in $A$ and $B$. Finally, we prove that the best proximity points $z \in A$ and $y \in B$ are unique. Assume that $z_{1}\left(\neq z_{2}\right) \in A$ are two distinct best proximity points of $S$ : $A \cup B \rightarrow A \cup B$ in $A$. Thus, $S z_{1}\left(\neq S z_{2}\right) \in B$ are two distinct best proximity points in $B$. Otherwise, $S z_{1}=S z_{2} \Rightarrow S^{2} z_{1}=S^{2} z_{2} \Rightarrow z_{1}=z_{2}$, since $z_{1}$ and $z_{1}$ are best proximity points, contradicts $z_{1} \neq z_{2}$. From Lemma $2.1(\mathrm{i})$ and $d\left(S z_{1}, S^{2} z_{2}\right)=d\left(S z_{2}, S^{2} z_{1}\right)=d\left(z_{1}, S z_{2}\right)=d\left(z_{2}, S z_{1}\right)=D$ through a similar argument to that concluding with (4.8) with the convexity of $A$ and the strict convexity of $(X,\|\|)$, guaranteed by its uniform convexity, one gets the following contradiction:

$$
D=d\left(S^{2} z_{1}, S z_{2}\right) \leq\left\|\frac{S^{2} z_{1}-S z_{1}}{2}\right\|+\left\|\frac{z_{2}-S z_{2}}{2}\right\|<\frac{D}{2}+\frac{D}{2}=D
$$

since $S^{2} z_{1}-S z_{1} \neq S z_{1}-z_{1}$. Thus, $z_{1}$ is the unique best proximity point of $S: A \cup B \rightarrow A \cup B$ in $A$ while $S z_{1}$ is its unique best proximity point in $B$.

Now, note that the condition $d(x, S x) \leq d(x, T x)$ applied to the best proximity points yields

$$
\begin{aligned}
D & =d(z, y)=d(z, S z)=d(y, S y) \leq d=d(y, S y) \\
& =d\left(z, T S^{2} z\right)=d\left(y, T S^{2} y\right)=d(y, T S z)=D,
\end{aligned}
$$

which implies strict equalities in (4.11), i.e.

$$
\begin{aligned}
D & =d(z, y)=d(z, S z)=d(y, S y)=d(z, T z)=d(z, T S y) \\
& =d\left(z, T S^{2} z\right)=d\left(y, T S^{2} y\right)=d(y, T S z)=D .
\end{aligned}
$$

If $A \cap B \neq \emptyset$, then $y=z$ is the unique fixed point of $S, T, T \circ S: A \cup B \rightarrow A \cup B$ from Theorem 2.3 .

In a similar way, Theorem 2.4 might be directly extended via Lemma 2.2 for the modification (2.9) of the contractive condition (2.1). On the other hand, Theorem 2.6 is extended via Lemma 2.5 under the contractive constraint (2.12) if, in general, $D \neq 0$ as follows.

Theorem 4.2 Let $(X,\|\|)$ be a uniformly convex Banach space and assume that $S, T$ : $A \cup B \rightarrow A \cup B$ are cyclic self-mappings satisfying the contractive condition (2.1), subject to the constraints $\min (\alpha, \beta, \gamma, \mu, \nu) \geq 0, \omega=1-\alpha-2(\beta+\gamma)$ and $\alpha+\delta+\beta \mu+\gamma \nu<1$ and $d(x, S x) \leq d(x, T x) ; \forall x \in A \cup B$, where the subsets $A$ and $B$ are nonempty, closed and convex. Then, there exist two unique best proximity points $z \in A, y \in B$ of the self-mappings $S, T, T$ 。 $S: A \cup B \rightarrow A \cup B$ such that (4.1)-(4.2) hold.

If $A \cap B \neq \emptyset$, then $z=y \in A \cap B$ is the unique fixed point of $S, T, T \circ S: A \cup B \rightarrow A \cup B$ which is in $A \cap B$. 
The proof of Theorem 4.2 is very similar to that of Theorem 4.1 by using Lemma 2.5 . In a similar way, Theorem 3.2 is extended as follows under Lemma 3.1 allowing the removal of the constraint $d(x, S x) \leq d(x, T x) ; \forall x \in A \cup B$. The proof is very close to that of Theorem 4.1 and is, therefore, omitted.

Theorem 4.3 Let $(X,\|\|)$ be a uniformly convex Banach space and assume that $S, T: A \cup$ $B \rightarrow A \cup B$ are cyclic self-mappings where $A$ and $B$ intersect and are nonempty and closed, and that the contractive condition (2.1) holds subject to the constraints $\min (\alpha, \beta, \gamma, \mu, \nu) \geq 0$ and

$$
\begin{aligned}
& 0 \leq \alpha<\frac{(1-\beta-\gamma)^{2}}{1+\beta-\gamma}-(\beta+\gamma) \\
& 0 \leq \beta<\min \left(\frac{1+\gamma(2 \gamma-3)}{3-2 \gamma}, 1-2 \gamma\right) ; \quad \gamma \in(1 / 2,1), \\
& \omega=\frac{(1-\beta-\gamma)^{2}-(1+\beta-\gamma)(\alpha+\beta-\gamma)}{2(1-\gamma)} .
\end{aligned}
$$

Then, there exist two unique best proximity points $z \in A, y \in B$ of the self-mappings $S, T, T$ 。 $S: A \cup B \rightarrow A \cup B$ such that (4.1)-(4.2) hold.

If $A \cap B \neq \emptyset$, then $z=y \in A \cap B$ is the unique fixed point of $S, T, T \circ S: A \cup B \rightarrow A \cup B$ which is in $A \cap B$.

It has to be pointed out that if $\omega$ in Theorems 4.1-4.3 is not given by the corresponding definitions but instead their respective equality right-hand sides are strict lower-bounds of $\omega$, then the distances in Lemmas 2.1, 2.2, 2.5 and 3.1 do not converge to $D$ but to some $\bar{D}>D$. The iterates $S^{2 n} x$ and $S^{2 n+1} x$ are always in $A$ and $B$ for any $x \in A$ and, respectively, in $B$ and $A$ for any $x \in B$, and they are as a result in some nonempty subsets $A^{\prime} \subseteq A$ and $B^{\prime} \subseteq B$ such that $\bar{D}:=\operatorname{dist}\left(A^{\prime}, B^{\prime}\right)>D$ or, conversely, as $n \rightarrow \infty$ by construction since $S(A) \subseteq B$, $T(A) \subseteq B, S(B) \subseteq A$ and $T(B) \subseteq A$. Lemmas 3.7 and 3.8 of [14] still hold. Then, $\left\{S^{2 n} x\right\}_{n \in \boldsymbol{N}}$ and $\left\{S^{2 n+1} x\right\}_{n \in \mathbf{N}}$ are Cauchy sequences which converge to some $z \in A$ and $S z \in B$ such that $d(z, S z)=\bar{D}$ if $x \in A$ and to $S z$ and $z$ if $x \in B$ which are unique since $A$ and $B$ are closed and convex and $(X,\|\|)$ is a uniformly convex Banach space. The sets $A^{\prime}$ and $B^{\prime}$ are non-unique but they are in families $\mathrm{A}^{\prime}$ and $\mathrm{B}^{\prime}$ of the subsets of $A$ and $B$ which contain by construction the two above unique convergence points. Then, the convergence points of the Cauchy sequences $z=S^{2} z \in A$ and $S z \in B$ are the unique best proximity points of all the closed convex sets in the families $\mathrm{A}^{\prime}$ and $\mathrm{B}^{\prime}$ of the subsets of $A$ and $B$ if $\bar{D}>D>0$. Then, Theorems 4.1-4.3 extend as follows.

Corollary 4.4 Assume that Theorems 4.1-4.3 are reformulated under respective identical assumptions except that $D>0$ and the respective definitions of $\omega$ are replaced with strict lower-bounds for their respective right-hand sides. Then, there exist two unique best proximity points $z=S^{2} z \in A$ and $S z=T z=T S^{2} z \in B$ of all sets in two families $\mathrm{A}^{\prime}$ and $\mathrm{B}^{\prime}$ of nonempty, closed and convex subsets of $A$ and $B$ which are convergence points of the sequences $\left\{S^{2 n} x\right\}_{n \in \boldsymbol{N}}$ and $\left\{S^{2 n+1} x\right\}_{n \in \boldsymbol{N}}$ for $x \in A$ so that $S x \in B$. 


\section{Examples}

Example 5.1 Define the discrete time-invariant scalar positive dynamic systems

$$
x_{n+1}=S x_{n}:=a x_{n}+b ; \quad y_{n+1}=T y_{n}:=c y_{n}+d ; \quad \forall n \in \mathbf{N}_{0},
$$

where $\boldsymbol{N}_{0}=\boldsymbol{N} \cup\{0\}$ with $a, c \in[0,1)$ and $\min (b, d) \geq 0$ subject to initial conditions satisfying $\min \left(x_{0}, y_{0}\right) \geq \frac{d-b}{a-c}$. The respective solutions converge asymptotically to the globally stable equilibrium points $x^{*}=\frac{b}{1-a}$ and $y^{*}=\frac{d}{1-c}$ which are both identical if $d=\frac{1-c}{1-a} b$ for $\min \left(x_{0}, y_{0}\right) \geq \frac{b}{1-a}$. Then, note also that $x^{*}=S x^{*}=\frac{a b}{1-a}+b=\frac{b}{1-a}$ and $y^{*}=T y^{*}=x^{*}=S x^{*}=$ $\frac{c d}{1-c}+d=\frac{b}{1-a}$ is a common unique fixed point of $S, T:\left[\frac{b}{1-a}, \infty\right) \rightarrow\left[\frac{b}{1-a}, \infty\right)$. This result also follows from Lemma 2.1, and Theorem 2.3 with $x_{n}, y_{n}\left(=x_{n}\right) \in A \equiv B:=\left[\frac{b}{1-a}, \infty\right) ; \forall n \in \mathbf{N}_{0}$ yields for $x=y \in A$ and $D=\operatorname{dist}(A, B)=0$ since $A \cap B=A \neq \emptyset$ under the contractive constraint (2.1):

$$
(1-\gamma-\beta) d\left(S x, S^{2} x\right) \leq(1-\gamma-\beta) d(S x, T S x) \leq(\alpha+\beta+\gamma) d(x, S x)
$$

if $\alpha+2(\beta+\gamma)<1$ from the necessary condition of Lemma 2.1

$$
d(x, S x) \leq d(x, T x) \quad \Rightarrow \quad d(S x, T S x) \geq d\left(S x, S^{2} x\right)
$$

Then, (5.2) is re-arranged as $d\left(S^{2} x, S x\right) \leq k d(S x, x)$ being contractive provided that

$$
k=\frac{\alpha+\beta+\gamma}{1-\beta-\gamma}<1 \quad \Leftrightarrow \quad \alpha+2(\beta+\gamma)<1
$$

since the necessary condition $d(x, S x) \leq d(x, T x) \Leftrightarrow x \geq \frac{d-b}{a-c}=x^{*} ; \forall x \in A$ holds for the Euclidean metric $d(x, y)=|x-y|$. Then, $S:\left[\frac{b}{1-a}, \infty\right) \rightarrow\left[\frac{b}{1-a}, \infty\right)$ is contractive, so that $d\left(S^{n+1} x_{0}, S^{n} x_{0}\right) \rightarrow 0$ as $n \rightarrow \infty$, and has a unique fixed point $x^{*}=y^{*}=\frac{b}{1-a} \in A(=A \cap B)$ since it is continuous. It also holds that $x^{*}$ is a fixed point of the composite mappings $T \circ S:\left[\frac{b}{1-a}, \infty\right) \rightarrow\left[\frac{b}{1-a}, \infty\right)$ (Theorem 2.3) and $S \circ T:\left[\frac{b}{1-a}, \infty\right) \rightarrow\left[\frac{b}{1-a}, \infty\right)$. Note that the necessary condition of Lemma $2.1 d(x, S x) \leq d(x, T x) \Leftrightarrow x \geq \frac{d-b}{a-c}=x^{*}$ justifies to fix $A \equiv B=\left[\frac{b}{1-a}, \infty\right)$ as definition domain of the self-mappings $S$ and $T$.

Example 5.2 The results of Example 5.1 also hold from Lemma 2.2 and Theorem 2.4 for the contractive constraint (2.9) subject to $\alpha+2 \beta+\gamma<1$.

Example 5.3 Consider the following dynamic systems:

$$
\begin{array}{lll}
x_{2 n+1}=S x_{2 n}:=a x_{2 n}+b ; & x_{2 n+2}=S x_{2 n+1}:=-x_{2 n+1} ; & \forall n \in \mathbf{N}_{0}, \\
y_{2 n+1}=T y_{2 n}:=a y_{2 n}+b ; & y_{2 n+2}=T y_{2 n+1}:=-y_{2 n+1} ; & \forall n \in \mathbf{N}_{0}
\end{array}
$$

under the same constraints of Example 5.1. Define real subsets $A:=\left[\frac{b}{1-a}, \infty\right)$ and $B:=$ $\left(-\infty, \frac{b}{a-1}\right]$ of empty intersection whose Euclidean distance is $D=\frac{2 b}{1-a} \geq 0$ and consider maps $S, T:\left(-\infty, \frac{b}{a-1}\right] \rightarrow\left(-\infty, \frac{b}{a-1}\right]$ being associated with the solutions of both dynamic systems which fulfil the necessary condition of Lemma $2.1 d(x, S x) \leq d(x, T x)$ everywhere in their definition domain. It follows the convergence to unique best proximity points $x^{*}$ 
being the limit of the sequences $\left\{x_{2 n}\right\}$ and $\left\{y_{2 n}\right\}$ and $z^{*}=-x^{*}$ being that of the sequences $\left\{x_{2 n+1}\right\}$ and $\left\{y_{2 n+1}\right\}$ if $x_{0}, y_{0} \in A$ and, conversely, if $x_{0}, y_{0} \in B$ which is a unique common fixed point $x^{*}=0$ of $S, T, T \circ S, T \circ S: \boldsymbol{R} \rightarrow \boldsymbol{R}$ if $b=d=0$ with $A \equiv B:=\boldsymbol{R}$. The conclusion also follows directly from Lemma 2.1, under the constraint (2.1), with $\lambda:=\alpha+2(\beta+\gamma)<1$ and $\omega=1-\lambda$, and Lemma 2.2, under the constraint (2.9) with $\alpha+2 \beta+\gamma<1$ and $\omega=1-\lambda$, and Theorem 4.1.

Example 5.4 The extension of the above examples to the non-scalar case is direct. For instance, consider the discrete dynamic systems:

$$
\begin{aligned}
& x_{n+1}=S x_{n}:=M x_{n}+m ; \quad \forall n \in \boldsymbol{N}_{0}, \\
& y_{n+1}=T y_{n}:=C_{n} y_{n}+G_{n} d ; \quad \forall n \in \boldsymbol{N}_{0},
\end{aligned}
$$

where $M(\neq 0), C_{n}(\neq 0) \in \boldsymbol{R}_{0+}^{p \times p}, m, G_{n} \in \boldsymbol{R}_{0+}^{p}, d \in \boldsymbol{R}_{0+}$ and the real sequences $\left\{C_{n}\right\},\left\{G_{n}\right\}$ are bounded. Assume that $M, G_{n}$ are convergent matrices for $n \in \boldsymbol{N}_{0}$ and that $\left\|\prod_{j=q}^{q+\ell-1}\left[C_{j}\right]\right\| \leq$ $K \rho^{\ell} ; \forall q \in \boldsymbol{N}_{0}, \forall \ell \in \boldsymbol{N}$ for some, in general, norm-dependent $K \in[1, \infty)$ and normindependent $\rho \in[0,1)$ real constants. Thus, $\left\{x_{n}\right\} \rightarrow x^{*}=(I-M)^{-1} m$ and $\left\{y_{n}\right\} \rightarrow y^{*}=$ $\sum_{i=0}^{\infty}\left(\prod_{j=i+1}^{\infty}\left[C_{j}\right] G_{i}\right) d$. Note that the dynamic systems (5.4)-(5.5) can be easily described in a close way for $G_{n} \in \boldsymbol{R}_{0+}^{p \times p}$ and $d \in \boldsymbol{R}_{0+}^{p}$. We can take the Euclidean norm (and metric) in $\boldsymbol{R}^{p}$ for the subsequent discussion and the corresponding vector-induced spectral matrix norm in $\boldsymbol{R}^{p \times p}$ which is compatible for well-posed mixed vector/matrix norm computations with the Euclidean vector norm. The fixed point $x^{*}=y^{*}=(I-M)^{-1} m=\sum_{i=0}^{\infty}\left(\prod_{j=i+1}^{\infty}\left[C_{j}\right] G_{i}\right) d$ exists for any $d \in \boldsymbol{R}_{0+}$ which satisfies

$$
\left(\begin{array}{l}
m \\
d
\end{array}\right) \in \operatorname{Ker}\left[-(I-M)^{-1}: \sum_{i=0}^{\infty}\left(\prod_{j=i+1}^{\infty}\left[C_{j}\right] G_{i}\right)\right],
$$

where $I$ denotes the $p$ th identity matrix, since the above null-space is nonempty which holds from Rouché-Froebenius theorem from linear algebra, since

$$
\operatorname{rank}\left[-(I-M)^{-1}: \sum_{i=0}^{\infty}\left(\prod_{j=i+1}^{\infty}\left[C_{j}\right] G_{i}\right)\right]=p
$$

holds from $(I-M)$ being non-singular, which implies the compatibility of the subsequent algebraic system of linear equations:

$$
\left[-(I-M)^{-1} \vdots \sum_{i=0}^{\infty}\left(\prod_{j=i+1}^{\infty}\left[C_{j}\right] G_{i}\right)\right]\left(b^{T} \vdots d^{T}\right)^{T}=0 .
$$

On the other hand, note that if $\left\|\sum_{i=0}^{\infty}\left(\prod_{j=i+1}^{\infty}\left[C_{j}\right] G_{i}\right) d\right\|<\infty$ and $M$ is critically stable (i.e. it is singular with at least one eigenvalue of modulus one, while it has no eigenvalue with modulus larger than one), then there are still non-unique common fixed points which are also stable equilibrium points of both mappings if

$$
m=(I-M)\left(\sum_{i=0}^{\infty}\left(\prod_{j=i+1}^{\infty}\left[C_{j}\right] G_{i}\right)\right) d=(I-M) y^{*}
$$


since the algebraic linear system $(I-M) x^{*}=(I-M) y^{*}=m$ is indeterminate compatible since

$$
\begin{aligned}
p & =\operatorname{rank}[I-M, m]=\operatorname{rank}\left[I-M,(I-M)\left(\sum_{i=0}^{\infty}\left(\prod_{j=i+1}^{\infty}\left[C_{j}\right] G_{i}\right)\right) d\right] \\
& =\operatorname{rank}([I-M \vdots I-M] \operatorname{Block} \operatorname{Diag}(I \vdots m)) \leq \operatorname{rank}(I-M \vdots I-M)=\operatorname{rank}(I-M)=p .
\end{aligned}
$$

A particular interesting case of both mappings having the same unique fixed point, so that both dynamic systems have the same stable equilibrium point being identical to such a fixed point, is when the second dynamic system is a perturbation of the first one considered to be the nominal one, that is $C_{n}=M+\tilde{C}_{n} ; d=G^{-1} m$ and $G_{n} d=\left(G+\tilde{G}_{n}\right) d=m+\tilde{G}_{n} d$ with $G, G_{n}$ being real square $p$-matrices, provided that the following equations have a solution in $G$ irrespective of the $p$-vector $m$ :

$$
x^{*}=(I-M)^{-1} m=\sum_{i=0}^{\infty}\left(\prod_{j=i+1}^{\infty}\left[C_{j}\right]\left(G+\tilde{G}_{i}\right)\right) G^{-1} m=\sum_{i=0}^{\infty}\left(\prod_{j=i+1}^{\infty}\left[C_{j}\right]\left(I+\tilde{G}_{i} G^{-1}\right)\right) m,
$$

which is

$$
G=\left[(I-M)^{-1}-\sum_{i=0}^{\infty}\left(\prod_{j=i+1}^{\infty}\left[C_{j}\right]\right)\right]^{-1}\left(\sum_{i=0}^{\infty} \prod_{j=i+1}^{\infty}\left[C_{j}\right] \tilde{G}_{i}\right)
$$

provided that the sequences $\left\{C_{n}\right\}$ and $\left\{\tilde{G}_{n}\right\}$ are such that $\left[(I-M)^{-1}-\sum_{i=0}^{\infty}\left(\prod_{j=i+1}^{\infty}\left[C_{j}\right]\right)\right]$ and $\sum_{i=0}^{\infty} \prod_{j=i+1}^{\infty}\left[C_{j}\right] \tilde{G}_{i}$ are non-singular.

The discussion of the existence of common fixed points from Lemma 2.1 and Theorem 2.3 under the constraint (2.1) imply

$$
d\left(S^{2} x, S x\right) \leq k d(S x, T S x) ; \quad k=\frac{\alpha+\beta+\gamma}{1-\beta-\gamma}<1
$$

provided that $d(x, S x) \leq d(x, T x) \Rightarrow d(S x, T S x) \geq d\left(S x, S^{2} x\right)$ leading together to the contractive condition $d\left(S^{2} x, S x\right) \leq k d(S x, x)$ for the self-mappings $S, T: A \rightarrow A$ with

$$
A \equiv B:=\left\{z=\left(z_{1}, z_{2}, \ldots, z_{p}\right) \in \boldsymbol{R}^{p}: z_{i} \geq e_{i}^{T}(I-M)^{-1} m\right\},
$$

where $e_{i}$ is the $i$ th unit Euclidean vector in $\boldsymbol{R}^{p}$ whose $i$ th component is unit provided that $\left\{G_{n}\right\}$ is such that

$$
\left.\sum_{i=1}^{p}\left[\left(\left(M_{i}^{T}-\mathbf{1}\right) x_{i n}+m_{i}\right)^{2}-\left(C_{i n}^{T}-\mathbf{1}\right) x_{i n}+G_{i n}^{T} d\right)^{2}\right] \leq 0 ; \quad \forall n \in \mathbf{N}_{0}
$$

for $x \in A$ satisfying the sequences (5.4) and (5.5), where $M_{i}^{T}, C_{i n}^{T}$ and $G_{i n}^{T} ; \forall n \in \boldsymbol{N}_{0}$ are the $i$ th row of the matrices $M, C_{n}$ and $G_{n}^{T} ; \forall n \in \boldsymbol{N}_{0}$ respectively; $x_{i n}$ and $m_{i}$ are the $i$ th components of $x_{n} ; \forall n \in \boldsymbol{N}_{0}$ and $m$, respectively; $\mathbf{1}$ is a Euclidean $p$-vector with all its components being one. It can be easily seen that (5.10) is equivalent to the necessary condition $\|(I-S) x\|=d(x, S x) \leq d(x, T x)=\|(I-T) x\|$ of Lemma 2.1 for the Euclidean metric. 


\section{Competing interests}

The authors declare that they have no competing interests.

\section{Authors' contributions}

Both authors contributed equally and significantly in writing this paper. Both authors read and approved the final manuscript.

\section{Author details}

${ }^{1}$ Instituto de Investigacion y Desarrollo de Procesos, Universidad del Pais Vasco, Campus of Leioa (Bizkaia), Aptdo. 644, Bilbao, Bilbao 48080, Spain. ${ }^{2}$ Department of Mathematics, Texas A\&M University- Kingsville, 700 University Blvd., Kingsville, TX 78363-8202, USA. ${ }^{3}$ Department of Mathematics, Faculty of Science, King Abdulazid University, Jeddah, 21589, Saudi Arabia.

\section{Acknowledgements}

The authors are grateful to the Spanish Ministry of Education for its partial support of this work through Grant DPI2009-07197. They are also grateful to the Basque Government for its support through Grants IT378-10 and SAIOTEK S-PE08UN15 and 09UN12. Finally, the authors are very grateful to the reviewers for their comments which have been very useful when improving the first version of the manuscript.

\section{Received: 14 May 2012 Accepted: 15 August 2012 Published: 31 August 2012}

\section{References}

1. Harjani, J, Lopez, B, Sadarangani, K: A fixed point theorem for mappings satisfying a contractive condition of rational type of partially ordered metric space. Abstr. Appl. Anal. 2010, Article Number 190701 (2010). doi:10.1155/2010/190701

2. Harjani, J, Sadarangani, K: Fixed point theorems for weakly contractive mappings in partly ordered sets. Nonlinear Anal., Theory Methods Appl. 71(7-8), 3403-3410 (2009)

3. Bhardwaj, R, Rajput, SS, Yadava, RN: Application of fixed point theory in metric spaces. Thai J. Math. 5(2), 253-259 (2007)

4. Enjouji, Y, Nakanishi, M, Suzuki, T: A generalization of Kannan's fixed point theorem. Fixed Point Theory Appl. 2009, Article Number 192872 (2009). doi:10.1155/2009/192872

5. Banach, S: Sur les opérations dans les ensembles abstracts et leur application aux équations intégrales. Fundam. Math. 3, 133-181 (1922)

6. Chatterjee, SK: Fixed point theorems. Comptes Ren. Acad. Bulgaria Sci. 25, 727-730 (1972)

7. Fisher, B: A fixed point theorem for compact metric spaces. Publ. Math. (Debr.) 25, 193-194 (1978)

8. Kannan, R: Some results on fixed points. Bull. Calcutta Math. Soc. 60, 71-76 (1968)

9. Kannan, R: Some results on fixed points-II. Am. Math. Mon. 76, 405-408 (1969)

10. Reich, S: Some remarks concerning contraction mappings. Canad. Math. Bull. 14, 121-124 (1971)

11. Kikkawa, M, Suzuki, T: Some similarity between contractions and Kannan mappings. Fixed Point Theory Appl. 2008, Article ID 649749 (2008). doi:10.1155/2009/649749

12. Subrahmanyam, PV: Completeness and fixed points. Monatsh. Math. 80(4), 325-330 (1975)

13. Kirk, WA, Srinivasan, PS, Veeramani, P: Fixed points for mappings satisfying cyclical contractive conditions. Fixed Point Theory 4(1), $79-89$ (2003)

14. Eldred, AA, Veeramani, P: Existence and convergence of best proximity points. J. Math. Anal. Appl. 323, 1001-1006 (2006)

15. Karpagam, S, Agrawal, S: Best proximity point theorems for $p$-cyclic Meir-Keeler contractions. Fixed Point Theory Appl. 2009, Article Number 197308 (2009). doi:10.1155/2009/197308

16. Di Bari, C, Suzuki, T, Vetro, C: Best proximity points for cyclic Meir-Keeler contractions. Nonlinear Anal., Theory Methods Appl. 69(11), 3790-3794 (2008)

17. De la Sen, M: Linking contractive self-mappings and cyclic Meir-Keeler contractions with Kannan self-mappings. Fixed Point Theory Appl. 2010, Article Number 572057 (2010). doi:10.1155/2010/572057

18. De la Sen, M: Some combined relations between contractive mappings, Kannan mappings, reasonable expansive mappings and T-stability. Fixed Point Theory Appl. 2009. Article Number 815637 (2009). doi:10.1155/2009/815637

19. Kikkawa, M, Suzuki, T: Three fixed point theorems for generalized contractions with constants in complete metric spaces. Nonlinear Anal., Theory Methods Appl. 69(9), 2942-2949 (2008)

20. Suzuki, T: Some notes on Meir-Keeler contractions and L-functions. Bull. Kyushu Inst. Technol., Pure Appl. Math. 53, 12-13 (2006)

21. Derafshpour, M, Rezapour, S, Shahzad, N: On the existence of best proximity points of cyclic contractions. Adv. Dyn. Syst. Appl. 6(1), 33-40 (2011)

22. Rezapour, S, Derafshpour, M, Shahzad, N: Best proximity points of cyclic $\varphi$-contractions on reflexive Banach spaces. Fixed Point Theory Appl. 2010, Article ID 946178 (2010). doi:10.1155/2010/046178

23. Al-Thagafi, MA, Shahzad, N: Convergence and existence results for best proximity points. Nonlinear Anal., Theory Methods Appl. 70(10), 3665-3671 (2009)

24. Qin, X, Kang, SM, Agarwal, RP: On the convergence of an implicit iterative process for generalized asymptotically quasi non-expansive mappings. Fixed Point Theory Appl. 2010, Article Number 714860 (2010). doi:10.1155/2010/7145860

25. Fisher, B: Results on common fixed points. Math. Jpn. 22, 335-338 (1977)

26. Nova, L: Puntos fijos comunes. Bol. Mat. (N.S.) IV, 43-47 (1997)

27. Ratchagit, M, Ratchagit, K: Asymptotic stability and stabilization of fixed points for iterative sequence. Int. J. Res. Rev. Comput. Sci. 2(4), 987-989 (2011)

28. Singh, SL, Mishra, SN, Chugh, R, Kamal, R: General common fixed point theorems and applications. J. Appl. Math. 2012, Article ID 902312 (2012). doi:10.1155/2012/902312

29. Chen, CM: Common fixed point theorems of the asymptotic sequences in ordered cone metric spaces. J. Appl. Math. 2011, Article ID 127521 (2011). doi:10.1155/20112/127521 
30. Chen, $\mathrm{CM}$, Chang, $\mathrm{TH}$ : Common fixed point theorems for a weaker Meir-Keeler type function in cone metric spaces. Appl. Math. Lett. 23(1), 1336-1341 (2010)

31. Huang, X, Zhu, C, Wen, X: Common fixed point theorems in fuzzy metric spaces using common EA property. J. Comput. Anal. Appl. 14(5), 967-973 (2012)

32. Abu-Donia, HM, Abd-Rabou, K: Common fixed point theorems for sequences of mappings under a new contractive condition of integral type. Appl. Math. Lett. 25(6), 980-985 (2012)

doi:10.1186/1687-1812-2012-136

Cite this article as: Sen and Agarwal: Common fixed points and best proximity points of two cyclic self-mappings.

Fixed Point Theory and Applications 2012 2012:136.

Submit your manuscript to a SpringerOpen ${ }^{\odot}$ journal and benefit from:

- Convenient online submission

- Rigorous peer review

Immediate publication on acceptance

Open access: articles freely available online

- High visibility within the field

- Retaining the copyright to your article

Submit your next manuscript at $>$ springeropen.com 